\title{
Chilling Stress Effects on Structure, Function and Development of Different Plant Processes
}

\section{Kashir Ali ${ }^{1 *}$, Muhammad Junaid Zaghum ${ }^{2}$, Zaman Ali' ${ }^{1}$, Muhammad Ussama Javaid $^{1}$, Muhammad Usman Qayyum ${ }^{1}$ and Ali Raza ${ }^{1}$}

${ }^{1}$ Institute of Soil and Environmental Sciences, University of Agriculture, Faisalabad

${ }^{2}$ Center for Excellence in Molecular Plant Sciences, Institute of Plant Physiology and

Ecology, Chinese Academy of Sciences, Shanghai, China

*Corresponding Author: Kashir Ali, Institute of Soil and Environmental Sciences, University of Agriculture, Faisalabad.
Received: January 03, 2022

Published: January 28, 2022

(C) All rights are reserved by Kashir Ali., et al.

\begin{abstract}
The unprecedented climate change has become a major issue around the globe. Abiotic stress which includes salt, drought, nutrient deficiency, pesticide contamination, light intensity as well as extreme low or high temperature inhibits or slow down many plant processes and ultimately cause the decreased or abnormal growth of the plant. These stresses reduce performance of four complex present in thylakoid membrane photosystem, cytochrome b6-f complex and ATP synthase. In chloroplasts, chilling stress may change the lipid membrane state and enzyme activity. The efficiency of photosynthesis then decreases, resulting in an overabundance of reactive oxygen species (ROS). There is a decline in antioxidant enzyme production, coupled with increased ROS accumulation in plants under environmental stress. A major negative effect has been observed on the activity of RuBisCo with increasing intensity of a range of environmental factors. The reduction in RuBisCo activity is due to the enzyme's activation state being downregulated in response to low temperature (e.g., by de-carbamylation and/or binding of inhibitory sugar phosphates). Chilling stress inhibits RuBisCo activation via a rapid and direct effect on RuBisCo activase. The present review tells how chilling stress can create serious effects on cellular membrane, biosynthesis of photosynthesis pigments, electron transport chain as well as RuBisCo activity.
\end{abstract}

Keywords: Chilling Stress; Low Temperature; Stress; Rubisco Activity; Environmental Stress

\section{Introduction}

Abiotic stress is the most common cause of crop failure, reducing the average yields of most main crops by more than $50 \%$ on average and endangering the long-term viability of agriculture around the globe. Temperature is one of the abiotic elements that has an impact on the development of plants. When plants are exposed to cold temperatures, they experience a loss in membrane fluidity, which has an impact on enzyme kinetic parameters and protein folding. As a result, developmental, morphological, physiological, and biochemical processes are disrupted, resulting in decreased yield, decreased quality, and decreased survival [1].
The production of ice inside plant cells may be very damaging. Even when the surrounding air temperature drops under 0 degrees Celsius, freeze-tolerant plants use a range of methods to limit the chance of this happening. Sustaining intracellular solute concentrations and stimulating ice nucleation outside the cells are two of these ways. The xerophytic adaptations that these plants have developed to cope with the decreased water availability both inside the plant and in the soil are also widespread. Temperatures as low as $5^{\circ} \mathrm{C}$ may kill a winter wheat plant that has not been hardened, despite its inherent ability to acclimate, stiffen, and acquire tolerance to freezing temperatures as low as $-20^{\circ} \mathrm{C}$. 
Carbon balance in plants is the primary factor influencing crop production. Plant productivity is measured by the difference between photosynthetic $\mathrm{CO}_{2}$ uptake and respiration rates. It is now well accepted that in vivo RuBisCo activity may be rapidly controlled in order to manage flow through the photosynthetic carbon reduction cycle in response to changes in the environment [2]. The enzyme ribulose-1,5-bisphosphate carboxylase/oxygenase (RuBisCo) is responsible for $\mathrm{CO}_{2}$ fixation during the photosynthetic process. It is predicted that plants fix more than 1011 tonnes of $\mathrm{CO}_{2}$ from the atmosphere each year, which has a significant impact on primary output.

Warm-climate plants' photosynthesis is considerably reduced when they are cooled. Tropical and subtropical species provide an excellent opportunity to investigate the effects of low temperature on photosynthetic processes that are not obscured by the abundance of protective responses observed in temperate species, thanks to the abundance of protective responses observed in temperate species. Low temperature, which is one of the most important abiotic stressors affecting the development and productivity of plants, severely depresses plant growth and results in a large decrease in grain output [3]. It causes a number of metabolic alterations, including the deactivation of numerous metabolic enzymes and the disruption of metabolic regulatory mechanisms, among other things [4], Osmolytes (e.g., proline and glycinebetaine) buildup [5], variations of glucose metabolism, and changes in photosynthetic characteristics are all possible outcomes [6].

Low temperature stress has a deleterious influence on a number of components of photosynthesis. It may, for example, impede thylakoid electron transport by increasing membrane viscosity and limiting plastoquinone diffusion, both of which are harmful to the process. It has been shown that low temperature stress has a negative effect on the activities of scavenging enzymes in the body [7]. As a result, the mitochondrial and chloroplastic electron transport processes are inefficient at counterbalancing the formation of ROS [8]. The review focuses on the effect of chilling stress on different plant processes like chloroplasts, RuBisCo activity, electron transport chain and photosynthetic efficiency as well as their structure, function and development.

\section{Effect of low temperature on chloroplast ultrastructure}

Low temperature is one of the abiotic variables that affects plant life and development. Low-temperature, or chilling, stress (damage produced by temperatures below freezing but above freezing) has been identified as a distinct environmental influence on agricultural plant physiology for more than 70 years. Many plant organisms experience physiological or cellular changes when exposed to low temperatures, which is referred to as low-temperature injury [9]. As a result, evolutionary, morphological, biochemical and physiological, mechanisms are influenced in many plants, influencing growth, efficiency, and survival [10].

While several variables determine the severity and duration of chilling damage, the ultrastructural consequences are almost identical across species. Chromatin condensation, lipid droplet aggregation, thylakoid dilatation, starch granule reduction, thylakoid swelling and disorganisation, chloroplast and mitochondrial swelling and disorder, and thylakoid swelling and disorganisation are some of the symptoms. Despite the fact that protective components (for example free proline, carbohydrates, and carotenoids) aggregate at low temperatures, a succession of detrimental repercussions will follow [11]. Typically, the most vulnerable targets are photosynthetic components, since their reaction is obvious as changes in pigment complexes, a fall in photosynthetic rates, loss of the chloroplast structure, or decreased electron transport and activity of enzymes [12]. The development of reactive oxygen species (ROS) is the most critical case when plants are subjected to low temperature.

Under favourable temperatures, chloroplasts have many starch granules and well-developed granal stacks that are intertwined by stromal thylakoids, and the chloroplast envelope's two membranes are intact. Chloroplast swelling, thylakoids deformation and swelling, and a reduction in the size and number of starch granules are often the first indicators of low temperature stress and the creation of tiny vesicles of the chloroplast membrane termed the peripheral reticulum. Additionally, low temperature stress might inhibit photosynthate transport out of the chloroplast. It is unknown if triose phosphate export from chloroplasts is prevented directly. Protein absorption by chloroplasts, which needs energy, is impaired in the cold owing to a deficient trans-envelope proton motive power [13]. As a result, another energy-intensive activity, photosynthate export from chloroplasts, is likely to be impeded as well. Indirectly, a reduction in phloem loading caused by cold may also limit photosynthate export [14]. It was also observed that the leaf soluble sugars can also rise during low temperature [15]. 




Figure 1: Effect of different levels of low temperature on chloroplast ultrastructure.

In cold temperatures, chloroplast pigments, including carotenoids and both chlorophylls, are impacted. Phenylpropanoid metabolism and flavonoid biosynthesis enzymes (phenylalanine ammonia-lyase enzymes) are stimulated by low ambient temperatures, resulting in an increased production of phenolic compounds that can tolerate stress conditions [16] recorded anthocyanin buildup was seen in maize leaves subjected to temperatures of $5^{\circ} \mathrm{C}$, $10^{\circ} \mathrm{C}$, and $15^{\circ} \mathrm{C}[17]$ and [18] noted elevated total phenolic content in the leaves of Lactuca sativa (exposed at $4{ }^{\circ} \mathrm{C}$ for 1 day) and Olea europaea (exposed at $4{ }^{\circ} \mathrm{C}$ for $12 \mathrm{~h}$ ).

In addition to their role in photoprotection, carotenoids serve as light pigments and ROS scavengers [19]. A major role is played by xanthophyll cycle pigments, a key subgroup of carotenoids, in the response to stress. When there is excess excitation energy that cannot be utilised for photosynthesis, the deep oxidation cycle converts violaxanthin into zeaxanthin and antheraxanthin. Low temperature stress has been associated to an increase in zeaxanthin concentration [20]. Reduced chlorophyll concentration indicates oxidative stress, as does a decrease in chlorophyll content after cooling. Low temperatures affected the pigment makeup of maize plants, resulting in a decrease in -carotene and an increase in zeaxanthin [21].

Cells of the mung bean that are susceptible to low temperatures show condensation and chromatin fragmentation [22]. Early observations (within 6 hours) indicated vesiculation of cellular membranes, including the ER and tonoplast, as well as vacuolation of plastids and mitochondria. Chilling damage proceeded in these cells in an orderly and predictable way. This was followed by the formation of enormous Golgi vesicles and parallel arrays of dilation of the endoplasmic reticulum (ER). Vesicles merged with vacuolar membranes and their contents were released after 72 - 96 hours of cytoplasm condensation. As the cooling progressed, the cytoplasm shrank away from the cell membrane, and organelles were digested, until the cell was digested completely. Because the cells were grown in culture, there were no physical signs to worry about.

\section{Decline in RuBisCo activity}

RuBisCo is biologically significant because its catalysis the main chemical reaction that allows inorganic carbon to release into the biosphere. One of the non-stomatal causes for the decline in photosynthesis rate was found to be a decrease in RuBisCo activity. Photosynthesis was impacted by a $90 \%$ fall in $\mathrm{O}_{2}$ content at cold temperatures $\left(16^{\circ} \mathrm{C}\right)$ because RuBisCo's activation state reduced at higher $\mathrm{CO}_{2}$ concentrations. Reducing the pressure gradient of ${ }_{\mathrm{C} 02}$ at cold temperatures increased the activation state of RuBisCo as well. It is necessary for the RuBisCo activation protein RuBisCo activase to be present. The rate at which ${ }_{\mathrm{CO} 2}$ is absorbed by plants depends heavily on the growth temperature [23].

As a general rule, plants that grow at relatively low temperature are more efficient in absorbing ${ }_{\mathrm{CO} 2}$ than plants that grow at normal room temperatures, but at high temperatures, they are less efficient. At roughly $17{ }^{\circ} \mathrm{C}$, the Arrhenius plot of RuBisCo activity shows a thermal split [24]. Because RuBisCo isn't being produced at low temperatures, photosynthesis suffers. The RuBisCo activase isoform is under redox regulation, and as a result, this enzyme becomes inactive during the hot midday and cool night [25].

At low temperatures, RuBisCo activation state recovers following $\mathrm{CO}_{2}$ decrease, which is linked to an indication of restrictions in RuBP regeneration. The $\mathrm{CO}_{2}$ saturation threshold of $\mathrm{C} 3$ and $\mathrm{C} 4$ photosynthesis falls below the present atmospheric $\mathrm{CO}_{2}$ levels below 20 degrees Celsius [26]. Stomatal closure is caused by a breakdown in the structure of guard cells, which influences the concentration of $\mathrm{CO}_{2}$ in the leaf, resulting in a drop-in RuBisCo activity [27]. Due to a decrease in oxygenase activity and decreased photorespiration, RuBisCo carboxylase activity should be controlled. Photorespiration, which reduces stomatal conductance in response to cooling, serves as a defense mechanism [28].

The photochemical efficiency of photosynthesis was considerably lowered when Phaseolus vulgaris was subjected to intense light and cooling temperatures [29]. Rice seedlings exposed to 
chilling stress produce more ROS, which raises the activity of the antioxidant catalase, suggesting that photorespiration plays a role in low temperature metabolism [30]. Due to slow $\mathrm{CO}_{2}$ fixation rate (kcat c, 2 - 3 s1) and competitive $\mathrm{O}_{2}$ fixation, which forms 2-phosphoglycolate and requires photorespiration recycling, the generation of RuBisCo is limited in C3 plants. Cold-treated seedlings had decreased levels of RuBisCo large subunits and RuBisCo binding protein, suggesting chloroplast injury.

\section{Effect on electron transport chain}

Temperature changes in plant mitochondria and chloroplast energy transducing membranes have attracted a lot of interest lately. Due to the adverse effects of low temperature stress on the photosynthetic electron transport system, photosynthesis is inhibited [31]. There are two large protein complexes in the thylakoid membrane known as photosystems I and II that employ incoming light energy to support a series of electron transfer processes. As early as the earliest biological research [32], cryogenic temperatures have been thoroughly studied in regard to this process.

In terms of a rate map, the temperature consequences on electron transport can be more readily visualized. In recent years the temperature has been increasingly influenced in the structure and operation of the energy-transducing membranes of plant mitochondria and chloroplasts. Most of this attention was based on the molecular comprehension of the cold sensitivity or chills of various plant species $[33,35]$.

A study of thylakoids extracted from midday leaves found that oxygen production was significantly reduced. This clearly shows that strong irradiance impacted leaf variations more severely on the decreasing side of PS2 than it did on CN leaves. Mitocryphal membranes obtained from cooling plants showed an increase in Ea' for respiratory enzymes and a decrease in lipid fluid at temperatures between 10 and $15 \mathrm{C}$, but not from cooling-resistant plants. $[33,35]$. PSII activity was strongly hindered and NPQ and photosynthetic electron transport were decreased when the shoots were chilled, but not the roots [36].

In plants from which mitochondria were extracted, the presence or absence of temperature-induced alterations to mitochondrial membranes is linked to cooling sensitivity and resistance, although studies from chloroplast membranes do not reveal an obvious relationship of this kind $[37,40]$. After removing or bypassing the rate-limiting processes, chloroplast membranes separated from both cold sensitive and cold resistant plants showed temperatureinduced Ea alterations [39]. There was a difference at a higher temperature not only in the chilling-temperature spectrum but in the chloroplast extracted from each plant.

If the major electron transfer process, photosynthetic linear transport, is hindered, then other mechanisms, like cyclic electron flow around PSI and chlororespiration, may be promoted [41], as plant health and survival are dependent on the ability to regulate photosynthetic electron transport. When the photosynthetic system is under low-temperature stress, these alternative ways of linear electron transport may assist to safeguard it [42].

Decrease photosynthetic efficiency of both photosystem (PS I and PS II)

Photosynthesis requires the activities related to electron transfer and energy capture. Photodamaging can occur here as a result of these reactions, which use redox chemistry and light intensity. A very sensitive to suboptimal environmental factors, as examples include the plant's geographic origin and to fluctuations of temperature, such as those that exist in the plants of the tropical or subtropical regions [43]. RuBP restoration lowers ${ }_{\mathrm{CO} 2}$ uptake in plants, which in turn reduces Rubisco activity, and this in turn reduces the rate of photosynthesis [44]. Photosynthesis's key activities, including as thylakoid electron transport, carbon reduction, and stomatal conductance control, would be impaired by these stimuli. Because NPQ (non-photochemical quenching) dispersion as heat of the excess energy suppresses photochemical performance at low temperatures, considerable levels of ROS accumulate in cells [45].

It is known that the PSII reaction center is affected when the architecture of related protein components is disrupted. It has been concluded that ROS does not actively trigger oxidative stress in the PSII operational centre but rather amplifies this photoinhibition by restricting protein synthesis, particularly the D1 protein, which is required for the PSII repair mechanism in the newly approved two-step system for PSII photoinhibition. ROS may be eliminated by plants' antioxidant systems, such as the water-water cycle [46]. The photosynthetic machinery may build up more free radicals as a result of cells becoming phototoxic when confronted with a cold discomfort [47]. 
PSII photoinhibition is minimal in chilling-sensitive plants such as cucumber (Cucumis sativus L.) and Arabidopsis thaliana when subjected to short-term chilling-light tension. This is especially true in cases when tropical tree species are exposed to chilly temperatures that are associated with modest light intensity [48]. Chilling-sensitive plants have different responses to cold temperatures in terms of PSII activity. As a result, nothing is known about the impact of these pressures on PSII behavior in the Paphiopedilum species.

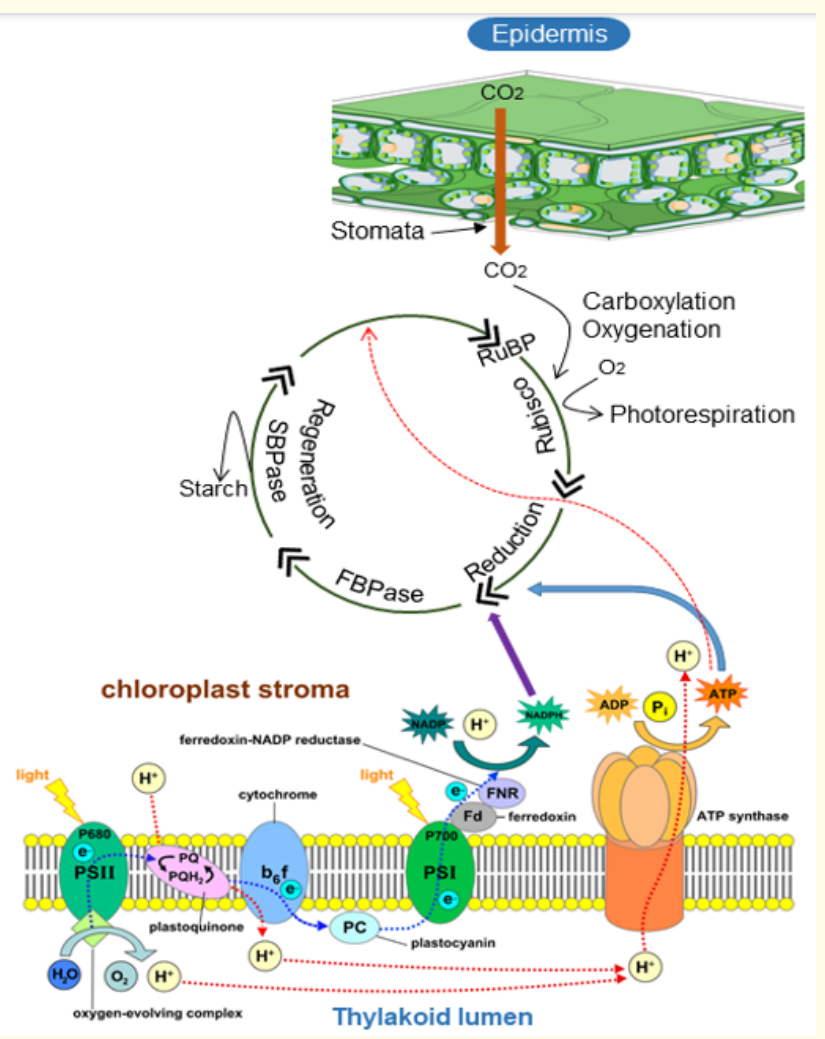

Figure 2: Effect of chilling stress on photosynthesis in thermophilic plants.

Weak electron transmission via linear electron few from PSII to PSI delays ${ }_{\mathrm{CO} 2}$ assimilation, resulting in hydroxyl radical generation and acceptor side over-reduction at PSI. This makes the PSI vulnerable to oxidative hydroxyl radicals at the electron acceptor end [49]. Tropical tree PSI activity is unaffected by cooling temperatures and moderate light intensity, despite the fact that PSI activity in sensitive cucumbers and Arabidopsis is reduced. PSI's performance varies from species to species, as is obvious [48].
Photoinhibition is a term used to describe the gradual and reversible limitation of photosynthetic activity caused by both harmful and regulating factors. A combination of stress elements, including bright light and heat, cold and dehydration, inhibits photosynthesis because plants absorb lighter than they utilise in photosynthetic energy [46]. The PSII photoinhibition site is wellknown. In chilling-sensitive and chilling-resistant species, PSII photoinhibition has been intensively researched for its detrimental and protective effects [50].

It has been believed that excessive irradiation in-vivo has little effect on PSI in general or has a negligible effect on it [27]. Even so, emerging research suggests that PSI can remain inhibited in response to light stress, especially at chilling temperatures $\left(0-10^{\circ} \mathrm{C}\right)$. When leaves of cold-sensitive cucumber (Cucumis sativus L.) and common bean (Phaseolus vulgaris L.) were cooled under low sunlight, photo-inhibition of PSI happened. When exposed to bright light, potato leaves (Solanum tuberosum L.) showed a considerable change [51]. Electron transport between PSII and PSI is required for PSI photoinhibition because of the need for molecular oxygen and PSI photo-inhibition. In the absence of oxygen, or even when diuron inhibited electron transmission from PSII to PSI, PSI inhibition was not seen. Active oxygen species (AOS) have been discovered to impede PSI [52].

Many thermophilic species are observed that if temperature getting low in dark and immediate chilling occurs then the photodamage process initiated. By contrast, the confluence of low temperature and high light can cause chronic photoinhibition of PSII [53]. Photorespiration and ${ }_{\mathrm{CO} 2}$ fixation are two examples of processes that utilise light as a source of excitation energy (light) but are slowed by lower temperatures. Smaller sinks for inhibitory energy used boost PSII's oxidative damage potential [54]. It is possible that decreasing fatty acid saturation by genetic alteration of thylakoid lipids might decrease high-light-low-temperature photoinhibition by enhancing diffusion and aiding repair even more. Additionally, light-chill-induced photosynthetic inhibition in thermophilic species is usually not the result of PSII photodamage [50].

Photosystem I (PSI) have been shown to be more effective at cooling than photosystem II (PSII) (PSII). In isolated thylakoid membranes, this is often evaluated using artificial electron donors or acceptors. PSI electron transport in intact plants may be measured using $820 \mathrm{~nm}$ absorption measures of the chlorophyll P700 
reaction cluster and the redox phase. [27]. This absorption of ${ }_{\mathrm{co} 2}$ has been researched under freezing circumstances to see what effects PSI, PSII, and other processes have.

Even though PSI activity declines more rapidly than PSII activity, this does not mean that PSI is the main target for relaxation. Due to the fact that improved PSI or PSII behaviour reported as a secondary response, the ultimate cold sensitive systems that have not been examined are not eliminated as a main target. As a consequence, complete leaf data is insufficient to pinpoint PSI as the principal focus of a terrifying set of events [47].

A drop in PSII quantum efficiency that can be reversed rapidly is essential for leaf photoprotection. Rearranging the xanthophyll pigments and creating an electrochemical potential variation in the transthylakoid molecule is a crucial defence against the more severe consequences of photodamage, as this procedure includes [55]. Detecting variations in the quenching of excitation energy in the antennae of PSII is straightforward owing to chlorophyll fluorescence [56]. This downregulation of PSII electron transport is frequently measured using quantum yields $\mathrm{Fv}^{\prime} / \mathrm{Fm}^{\prime}$ (excitation energy transfer efficiency to accessible PSII reaction centres and non-photochemical quenching) [57].

Heat is released from the reaction Centre when photosynthetic sinks cool down (simultaneously with or after cooling). This occurs because the light accumulation complex's transmission efficiency into the reaction centre is reduced. The thermophilic crops like maize and tomato, when exposed to light and cold, exhibit these phenomena. Tomatoes and mangoes, for example, don't have complicated photoinhibition to blame for their decline in photosynthesis after a frost [52].

It is fascinating to see some impacts of chilling on photosynthesis induced by failing to execute some circadian rhythms and the implications of chilling on carbohydrate metabolites can be underlined [47]. Light energy cooling has the potential to affect PSII photo-damage as well as redox modulation of stromal bisphosphatases, FBPase, SBPase, and presumably rubisco activase, although these effects are less apparent [58].

\section{Conclusion}

Many species, including essential agricultural crops, have productivity and geographic spread limitations because of low temperatures. Nonfreezing temperatures $\left(0-12^{\circ} \mathrm{C}\right)$ are typical throughout the growing season in temperate countries and may significantly reduce plant production, which is discussed here. Low temperature, which is one of the most important abiotic stresses impacting plant development and productivity, slows plant growth and reduces grain yield significantly. Photosynthetic transport out of the chloroplast is hindered by low temperature stress. Chilling stress obstructs thylakoid electron transport by increasing membrane viscosity and restricting plastoquinone diffusion, both of which are detrimental to the process. A reduction in RuBisCo activity was discovered to be one of the non-stomatal explanations of the fall in photosynthesis rate. Because of cells becoming phototoxic when faced with chilling stress the photosynthetic machinery may store up more free radicals. The effects of chilling on photosynthesis generated by failure to execute specific circadian cycles are significant, and the consequences of chilling on carbohydrate metabolites cannot be emphasized. In general, low temperature or chilling stress reduce development of chloroplast ultrastructure, RuBisCo activity, disturb electron transport chain and one of the major abiotic stresses in reduction of photosynthesis.

\section{Conflict of Interest}

The authors declare no conflict of interest.

\section{Bibliography}

1. C Vergnolle., et al. "The cold-induced early activation of phospholipase $\mathrm{C}$ and $\mathrm{D}$ pathways determines the response of two distinct clusters of genes in Arabidopsis cell suspensions". Plant Physiology 139.3 (2005): 1217-1233.

2. J Galmés., et al. "Variation in Rubisco content and activity under variable climatic factors". Photosynthesi Research 117.1-3 (2013): 73-90.

3. K Kosová., et al. "Proteome analysis of cold response in spring and winter wheat (Triticum aestivum) crowns reveals similarities in stress adaptation and differences in regulatory processes between the growth habits". Journal of Proteome Research 12.11 (2013): 4830-4845.

4. J Xu., et al. "Comparative physiological and proteomic response to abrupt low temperature stress between two winter wheat cultivars differing in low temperature tolerance". Plant Biology 15.2 (2013): 292-303.

5. P Thakur and H Nayyar. "Facing the cold stress by plants in the changing environment: sensing, signaling, and defending mechanisms". In Plant Acclimation to Environmental Stress, Springer (2013): 29-69. 
6. C Crosatti., et al. "Harden the chloroplast to protect the plant". Physiologia Plantarum 147.1 (2013): 55-63.

7. X Li., et al. "Induction of chilling tolerance in wheat during germination by pre-soaking seed with nitric oxide and gibberellin". Plant Growth Regulation 71 (2013): 31-40.

8. E Ruelland., et al. "Chapter 2 Cold Signalling and Cold Acclimation in Plants". 1st ed 49.C. Elesvier Ltd (2009).

9. JFHD Evers., et al. "Compared responses of poplar cuttings and in vitro raised shoots to short-term chilling treatments". Plant Cell Reports 19 (2000): 954-960.

10. P Maestrini., et al. "Isolation and expression analysis of low temperature-induced genes in white poplar (Populus alba)". Journal of Plant Physiology 166.14 (2009): 1544-1556.

11. J Renaut., et al. "Biochemical and physiological mechanisms related to cold acclimation and enhanced freezing tolerance in poplar plantlets". Physiologia Plantarum 125.1 (2005): 82-94.

12. CO Marian., et al. "Dehydrin variability among rhododendron species: A 25-kDa dehydrin is conserved and associated with cold acclimation across diverse species". New Phytologist 161.3 (2004): 773-780.

13. EA Leheny and SM Theg. "Apparent Inhibition of Chloroplast Protein Import by Cold Temperatures Is Due to Energetic Considerations Not Membrane Fluidity". Plant Cell 6.3 (1994): 427-437.

14. YV Gamalei., et al. "Effects of temperature on the conformation of the endoplasmic reticulum and on starch accumulation in leaves with the symplasmic minor-vein configuration". Planta 194.4 (1994): 443-453.

15. Å Strand., et al. "Development of Arabidopsis thaliana leaves at low temperatures releases the suppression of photosynthesis and photosynthetic gene expression despite the accumulation of soluble carbohydrates". The Plant Journal 12.3 (1997): 605614.

16. PJ Christie., et al. "Impact of low-temperature stress on general phenylpropanoid and anthocyanin pathways: Enhancement of transcript abundance and anthocyanin pigmentation in maize seedlings". Planta 194.4 (1994): 541-549.

17. MM Oh., et al. "Environmental stresses induce health-promoting phytochemicals in lettuce". Plant Physiology and Biochemistry 47.7 (2009): 578-583.
18. A Cansev., et al. "Alterations in total phenolic content and antioxidant capacity in response to low temperatures in olive (Olea Europaea L. 'Gemlik')”. Plant Arch 12.1 (2012): 489-494.

19. R Esteban., et al. "Internal and external factors affecting photosynthetic pigment composition in plants: A meta-analytical approach". New Phytologist 206.1 (2015): 268-280.

20. M Tausz., et al. "Biochemical responses in leaves of two apple tree cultivars subjected to progressing drought". Journal of Plant Physiology 162 (2005): 1308-1318.

21. P Haldimann. "Effects of changes in growth temperature on photosynthesis and carotenoid composition in Zea mays leaves". Physiologia Plantarum 97.3 (1996): 554-562.

22. HA Ishikawa. "Ultrastructural features of chilling injury: injured cells and the early events during chilling of suspensioncultured mung bean cells". American Journal of Botany 83.7 (1996): 825-835.

23. RF Sage and DS Kubien. "The temperature response of C3 and C4 photosynthesis". Plant Cell Environment 30.9 (2007): 10861106.

24. YP Cen and RF Sage. "The regulation of Rubisco activity in response to variation in temperature and atmospheric CO2 partial pressure in sweet potato". Plant Physiology 139.2 (2005): 979-990.

25. N Zhang and AR Portis. "Mechanism of light regulation of Rubisco: A specific role for the larger rubisco activase isoform involving reductive activation by thioredoxin-f". Proceedings of the National Academy of Sciences of the United States of America 96.16 (1999): 9438-9443.

26. RF Sage. "Variation in the kcat of Rubisco in C3 and C4 plants and some implications for photosynthetic performance at high and low temperature". Journal of Experimental Botany 53.369 (2002): 609-620.

27. DJ Allen and DR Ort. "Impacts of chilling temperatures on photosynthesis in warm-climate plants". Trends in Plant Science 6.1 (2001): 36-42.

28. I Voss., et al. "Emerging concept for the role of photorespiration as an important part of abiotic stress response". Plant Biology 15.4 (2013): 713-722.

29. SB Powles., et al. "Interaction between light and chilling temperature on the inhibition of photosynthesis in chilling-sensitive plants". Plant Cell Environment 6.2 (1983): 117-123. 
30. C Cheng., et al. "An early response regulatory cluster induced by low temperature and hydrogen peroxide in seedlings of chilling-tolerant japonica rice". BMC Genomics 8 (2007): 1-18.

31. X Li., et al. "Cold priming drives the sub-cellular antioxidant systems to protect photosynthetic electron transport against subsequent low temperature stress in winter wheat". Plant Physiology and Biochemistry 82 (2014): 34-43.

32. BYW Arnold and RK Clayton. "carotenoidless11 (obtained Sistrom) light” 46 (1960): 769-776.

33. JM Lyons. "Chilling Injury in Plants". Annual Review of Plant Biology 24.1 (1973): 445-466.

34. PJ Quinn and WP Williams. "Plant lipids and their role in membrane function” 34 (1978): 109-173.

35. JK Raison. "Biochemical explanation of low-temperature stress in tropical and sub-tropical plants". Bull R Soc NZ (1974).

36. K Suzuki., et al. "High root temperature blocks both linear and cyclic electron transport in the dark during chilling of the leaves of rice seedlings". Plant and Cell Physiology 52.9 (2011): 1697-1707.

37. H Inoué. "Break points in arrhenius plots of the hill reaction of spinach chloroplast fragments in the temperature range from -25 to $25^{\circ} \mathrm{C}^{\prime \prime}$. Plant and Cell Physiology 19.3 (1978): 355-363.

38. P Jursinic and Govindjee. "Temperature Dependence of Delayed Light Emission in the 6 to 340 Microsecond Range After a Single Flash in Chloroplasts". Photochemistry and Photobiology 26.6 (1977): 617-628.

39. WG Nolan and RM Smillie. "Temperature-induced Changes in Hill Activity of Chloroplasts Isolated from Chilling-sensitive and Chilling-resistant Plants". Plant Physiology 59.6 (1977): 1141-1145.

40. RM Smillie., et al. "Effect of Growth Temperature on Chloroplast Structure and Activity in Barley". Plant Physiology 62.2 (1978): 191-196.

41. D Rumeau., et al. "Chlororespiration and cyclic electron flow around PSI during photosynthesis and plant stress response". Plant Cell Environment 30.9 (2007): 1041-1051.

42. MJ Quiles. "Regulation of the expression of chloroplast $n d h$ genes by light intensity applied during oat plant growth". Plant Science 168.6 (2005): 1561-1569.
43. P Pospíšil. "Production of reactive oxygen species by photosystem II". Biochimica et Biophysica Acta - Bioenergetics 1787.10 (2009): 1151-1160.

44. W Huang., et al. "Moderate photoinhibition of photosystem II Protects Photosystem I from photodamage at chilling stress in tobacco leaves". Frontiers in Plant Science 7 (2016): 1-9.

45. Z Chen and DR Gallie. "Dehydroascorbate reductase affects non-photochemical quenching and photosynthetic performance". Journal of Biological Chemistry 283.31 (2008): 2134721361.

46. K Sonoike. "The different roles of chilling temperatures in the photoinhibition of photosystem I and photosystem II". Journal of Photochemistry and Photobiology B: Biology 48.2-3 (1999): 136-141.

47. JJ Eaton-Rye and R Sobotka. "Editorial: Assembly of the photosystem II membrane-protein complex of oxygenic photosynthesis". Frontiers in Plant Science 8 (2017): 1-4.

48. Z Zhang., et al. "The higher sensitivity of PSI to ROS results in lower chilling - light tolerance of photosystems in young leaves of cucumber". Journal of Photochemistry and Photobiology B: Biology 137 (2014): 127-134.

49. YJ Yang., et al. "The effects of chilling-light stress on photosystems I and II in three Paphiopedilum species". Botanical Studies 58.1 (2017): 53.

50. S Govindachary., et al. "Photosystem II inhibition by moderate light under low temperature in intact leaves of chilling-sensitive and -tolerant plants". Physiologia Plantarum 121.2 (2004): 322-333.

51. XG Li., et al. "The susceptibility of cucumber and sweet pepper to chilling under low irradiance is related to energy dissipation and water-water cycle". Photosynthetica 41.2 (, 2003): 259-265.

52. W Wang., et al. "Achieving solar overall water splitting with hybrid photosystems of photosystem II and artificial photocatalysts". Nature Communications 5 (2014): 4647.

53. F Michoux., et al. "Crystal structure of CyanoQ from the thermophilic cyanobacterium Thermosynechococcus elongatus and detection in isolated photosystem II complexes". Photosynthesis Research 122.1 (2014): 57-67.

54. Y Lima-Melo., et al. "Consequences of photosystem-I damage and repair on photosynthesis and carbon use in Arabidopsis thaliana". The Plant Journal 97.6 (2019): 1061-1072. 
55. C Deng., et al. "Toxic effects of mercury on PSI and PSII activities, membrane potential and transthylakoid proton gradient in Microsorium pteropus". Journal of Photochemistry and Photobiology B: Biology 127 (2013): 1-7.

56. U Schreiber and C Klughammer. "Evidence for variable chlorophyll fluorescence of photosystem I in vivo". Photosynthesis Research 149.1-2 (2021): 213-231.

57. Ö Turan and Y Ekmekçi. "Activities of photosystem II and antioxidant enzymes in chickpea (Cicer arietinum L.) cultivars exposed to chilling temperatures". Acta Physiologiae Plantarum 33.1 (2011): 67-78.

58. S Han., et al. "CO2 assimilation, photosystem II photochemistry, carbohydrate metabolism and antioxidant system of citrus leaves in response to boron stress". The Plant Journal 176.1 (2009): 143-153.

\section{Assets from publication with us}

- Prompt Acknowledgement after receiving the article

- Thorough Double blinded peer review

- Rapid Publication

- Issue of Publication Certificate

- High visibility of your Published work

Website: www.actascientific.com/

Submit Article: www.actascientific.com/submission.php

Email us: editor@actascientific.com

Contact us: +919182824667 